\title{
1. Understanding economics through the history of sport
}

\section{John K. Wilson}

Many people believe that interest in history has waned. University offerings of history degrees have fallen, and there are constant calls to reform and reinvent the teaching of history in our schools. While this may be to some extent true, it is interesting to observe that the sense of history held by most sports fans is deep, and has certainly not changed in many decades. Teams are remembered for their memorable clashes and rivalries, achievements, and on occasion, even their demise over time. Players even long dead are remembered, discussed and compared against their contemporaries. There is a fondness for old stadiums, leagues and these are constantly used as a benchmark against which modern sports are measured.

Emanating from such an interest in professional sports is a comprehensive documentation of achievements, attributes of players, leagues, major events, and other associated details through time. These data transcend the direct history of sport. They also have much to say about how societies function, how economies work and how changes have occurred.

Sports history provides a rich set of information and lessons for economists. Sport operates, in many ways, as a peculiar model in economics. Labour markets are often distorted, by policy or market power. Governments have pervasive influence over infrastructure related to sport, and governing bodies enjoy some exemptions from regulations that are commonplace in other sectors of the economy. These peculiarities are the lens through which sports economics can illuminate the underlying economic issues that pervade society. When a salary cap is implemented, for example, we get a chance to observe a natural experiment that can shed light on the operation of all labour markets.

This volume builds on earlier work published in Sports Through the Lens of Economic History. The papers contained here, like in our earlier volume, provide insights not only into sport, but a much broader world around us.

The business of sport is significant. There are large rents associated with the operation of sporting teams and associated merchandising. Economic activity surrounding these activities is argued by governments to be sufficiently large that subsidies are warranted. This of course, was not always so. Indeed, most 
professional sporting entities began as amateur, recreational clubs. Exactly what precipitated the change from recreational status to these professional entities is the subject of Chapter 2. Dewhirst and Vamplew analyse this transition using the case studies of Queens Park and Bradford football clubs, both pioneers of commercialization in Britain. They develop a framework under which the process can be viewed, examining environmental factors (changing demographics and urban characteristics), internal changes within these clubs and organizational review and embrace of change that facilitated a more commercial stance.

Stadiums represent a large cost for sporting clubs, and often taxpayers. Chapters 3 and 4 examine some specific issues surrounding stadium development. In Chapter 3, Pomfret and Wilson look at the history of ground sharing. In many instances, rival clubs have almost equivalent facilities within walking distance of each other. The authors ask why, given the large costs, more ground sharing is not observed. They also investigate the changes to ground sharing arrangements that have occurred over time.

Frost, Borrowman, Mishra and Halabi examine the relationship between crowd attendance, competitive balance and stadium construction in the Scottish and English football leagues prior to the Second World War. They reveal a complex set of associations, with factors other than competitive balance as potential determinants of crowd attendance. This is particularly the case for certain clubs, where traditional rivalries existed. While difficult to affirm causation, their analysis suggests a relationship between stadium construction and attendance, and the authors discuss underlying forces that may explain this.

Chapters 5 and 6 explore mega-events through the perspective of history. Matheson (Chapter 5) focuses on the economic drivers surrounding the summer Olympics, and in particular documents the long-run history of bidding. He identifies periods where bidding is intense, but also where the number of bidders falls dramatically. For example, he identifies three periods of declining host interest: the first following Nazi extravagance in 1936, the second following terrorism in Munich (1972) the third after and cost overruns in Montreal (1976). Profitability of the games is shown to increase interest post Los Angeles but in recent years, cost pressures are again seeing a waning interest in (particularly) developed nations hosting the games.

Andreff (Chapter 6) provides a comprehensive overview of issues underlying economic impact studies of mega events in general. One key aspect is that the estimation of multiplier effects turns crucially on the state of the economy when the event is held. This is of particular interest given the lag between bidding for and winning hosting rights and actually hosting the event is sometimes large. Andreff cites several cases where a country's bid has taken place during a period of good economic conditions but actual hosting has 
occurred during a recession. He also points to the related winter sports product sector, and identifies the importance of product life cycles. In doing this, he brings together two geographically close case studies held at differing periods (Grenoble in 1968 and Albertville in 1992).

In many cases sports are played with identical, or at least similar rules across countries. This represents a unique opportunity to examine the influences of different cultures, political systems, and economic conditions within the context of an homogenous good between countries. Kawaura and La Croix (Chapter 7) use baseball in Japan and the USA to conduct just such an analysis. They uncover a pattern of differing delegation patterns by team owners towards their league commissioners.

Broader social patterns are investigated in Chapters 8 (Price, Brown and Van Leeuwen) and 9 (Ross, Cranfield and Inwood). For a large cohort of players born before 1940, Price et al. explore the effects of a playing career in baseball on longevity. This provides an interesting insight into what have become strong contemporary debates surrounding the risks associated with professional sports, whether through injury or other factors that may adversely affect life outcomes due to an early, but relatively short-lived career in sport. Their results suggest that playing major (and to some extent minor) league baseball is positively associated with life expectancy. Ross et al. track attributes of players in the Canadian hockey labour market in terms of their stature. They show that players have generally shown an increase in height, weight and BMI across the twentieth century. Interestingly, their results are positioned against the general population and it is shown that the 'athletic premium' remained fairly constant over the period. They also find, however, that there are differences between positions and also that the athletic premium has been influenced at times by rule changes favouring shorter players.

Leeds and Rockoff (Chapter 10) deal with the issue of prejudice against African American riders in the Kentucky Derby between 1870 and 1911. Because horse racing has measurable reward structures and performance measures, it is very suited to examining this issue. Their results reveal that African American jockeys were more likely to finish in a higher position than was predicted by betting odds. One interpretation offered is that punters were in some sense prepared to bet against these jockeys for non-pecuniary reasons that may reveal some prejudice. However they also note that there were still owners willing to provide rides for them and betting markets to support them.

A more general history of sports betting is provided by Pradier in Chapter 11. He traces the long run history of sports gambling and links to economic theories and explains a variety of changes over time, ranging from peer-to-peer betting through to global online systems.

Finally, the volume is completed with an analysis of survival of clubs in the English Premier League. This league has retained an open system of pro- 
motion and relegation, where the financial stakes of being relegated are very large. Chang, Borrowman and Frost use data from the league's inception and determine that club survival in the league to be a function of revenue, foreign player composition, previous relegation history and manager quality. 